\title{
Comparación de la efectividad de la dosis única frente a la dosis triple de cefazolina como profilaxis antibiótica en cesáreas
}

Pedro Ochoa Allemant ${ }^{1}$, Evelyn Obregón Julca ${ }^{1}$, Rodrigo Núñez Bravo${ }^{1}$, Fiorella Núñez del Prado Zora Carbajal ${ }^{1}$, Cristina María Ojeda Nieto ${ }^{1}$

\section{RESUMEN}

Objetivo: Determinar la efectividad de la dosis única comparada con la dosis triple de Cefazolina como profilaxis antibiótica para reducir las infecciones post-cesárea.

Material y Métodos: Estudio observacional, analítico, retrospectivo, transversal y comparativo. Se incluyó 82 historias clínicas de pacientes sometidas a cesárea en el Hospital Nacional Guillermo Almenara Irigoyen que recibieron dosis única o dosis triple de Cefazolina en el año 2011. De acuerdo al esquema recibido,se evaluaron las complicaciones infecciosas. Resultados: No hubo diferencia estadísticamente significativa entre la dosis única y la dosis triple en la incidencia de infecciones totales $(9,3 \%$ vs $16,4 \%, p=0,422)$, infección del sitio operatorio ( $7 \%$ vs $12,8 \%, p=0,793)$, endometritis $(0 \%$ vs $2,6 \%, p=1,116)$ e infección del tracto urinario $(2,3 \%$ vs $0 \%, p=0,918)$.

Conclusión: La dosis única de Cefazolina como profilaxis antibiótica fue igual de efectiva como la dosis triple en la prevención de la morbilidad infecciosa post-cesárea. (Horiz Med 2014; 14(3): 37-43)

Palabras clave: Cefazolina, profilaxis antibiótica, cesárea, infección post-cesárea. (Fuente: DeCS BIREME).

Comparison of single versus triple dose of cefazolin in antibiotic prophylaxis for caesarean section

\section{ABSTRACT}

Objective: To compare the effectiveness of single dose versus triple dose of Cefazolinas prophylactic antibiotics in women undergoing cesarean section.

Material and Methods: An observational, analytic, retrospective, cross sectionalcomparative study. Eighty two medical records of women undergoing caesarean section who received either single dose or triple dose in the Almenaralrigoyen Hospital were included. Women were compared on the basis of development of postoperative infections.

Results: The study groups did not have statistically significant differences in the incidence of total infections $(9,3 \%$ vs $16,4 \%$, $p=0,422)$, wound infections ( $7 \%$ vs $12,8 \%, p=0,793)$, endometritis ( $0 \%$ vs $2,6 \%, p=1,116)$ and urinary tract infection $(2,3 \%$ vs $0 \%, p=0,918$ )

Conclusion: A single prophylaxis dose of Cefazolin was as effective as triple dose in preventing post-caesarean infectious morbidity. (Horiz Med 2014; 14(3): 37-43)

Key words: Cefazolin,antibiotic prophylaxis, caesarean section, post-caesarean infection. (Source: MeSH NLM).

1 Médico - Cirujano, Egresado de la FMH de la Universidad de San Martin de Porres. Lima, Perú 


\section{INTRODUCCIÓN}

El parto por cesárea constituye el factor de riesgo más importante de infección materna puerperal $(1,2)$. Comparado con el parto vaginal, el riesgo de infección es entre 5 a 20 veces mayor (3), llegando la incidencia de sepsis puerperal al 36\% (4). En la actualidad, la tasa de cesáreas a nivel mundial es del $15 \%$ (5) y en la mayoría de países de Latinoamérica es del $35.4 \%$ (6) siendo sus complicaciones infecciosas no solo una de las causas fundamentales de morbilidad materna sino también de estancia hospitalaria prolongada lo que representa un incremento en los costos de salud.

Existe evidencia clara que apoya el beneficio de los antibióticos profilácticos a quienes se les realiza una cesárea (7). Sin tratamiento profiláctico, la incidencia de endometritis oscila entre el $20 \%$ y el $85 \%$, y las tasas de infección de la herida operatoria y de complicaciones infecciosas graves pueden alcanzar hasta un $25 \%$ (8).

En cambio, con su uso, se puede llegar a reducir hasta un $75 \%$ la incidencia de las infecciones postoperatrias (9) tales como fiebre, infección del sitio operatorio, endometritis, bacteriemia, infección urinaria y otros tipos de infecciones graves (absceso pélvico, shock séptico, fascitis necrotizante $y$ tromboflebitis séptica de la vena iliaca). No obstante, aún existen temas controversiales tales como el agente profiláctico ideal, el momento de administración y el número de dosis.

En lo referente al antibiótico ideal, tanto Cefazolina (1gr) como Ampicilina (2gr), además de tener la misma efectividad (10), han demostrado ser superiores a otros esquemas (11), por lo que en la práctica clínica pueden utilizarse indistintamente.

Se ha demostrado que tanto la administración pre-incisional como la post-clampaje del cordón umbilical reducen significativamente la infección materna (7). La profilaxis antibiótica pre-incisional es la estandarizada para la gran mayoría de procedimientos quirúrgicos por lo que también debería serlo para las cesáreas (12).
En una revisión de varios estudios, se demostró que la dosis única era igual de efectiva que la dosis múltiple (13). Pese a ello, aun no se ha establecido el régimen de dosis estándar y se sigue empleando la dosis triple en nuestro medio.

En la revisión Cochrane publicada en 1999 (13), de 51 estudios clínicos randomizados, se concluyó que un régimen profiláctico con múltiples dosis no ofrece beneficio adicional en comparación con un tratamiento de dosis única; OR: 0.92 (IC 95\%: 0.70 a 1.23). Además, se menciona que los tratamientos con dosis única generalmente son menos costosos y podrían asegurar el uso generalizado de profilaxis antibiótica para lascesáreas, especialmente en países en vías de desarrollo.

La Society of Obstetricians and Gynaecologists of Canada (SOGC) en su Clinical Practice Guideline del 2010 (14), recomienda el uso de una sola dosis de cefalosporina de primera generación salvo en las pacientes con antecedentes de alergia a la penicilina donde sugiere Clindamicina 0 Eritromicina (I-A). Este antibiótico también fue recomendado en la Guía de 1999 de Centers for Disease Control and Prevention(CDC) (15) donde sugieren la administración endovenosa de 1 a 2 gramos en los 30 minutos previos a la incisión. Una dosis adicional puede ser considerada solo en el caso de pérdidas sanguíneas mayores a $1500 \mathrm{ml}$ y si es que el procedimiento dura más de 4 horas (16).

Igualmente, The Clinical Indication User Manual 2012 (Obstetric v7) de la Australian Council on Health care Standards (ACHS) (17)y The Royal Australian and New Zealand College of Obstetricians and Gynaecologists (RANZCOG) (18) 1gr de Cefazolina (EV) inmediatamente después del clampaje del cordón.

Otros estudios comoeldeGallónetal(19)demostraron que no hay diferencia significativamente estadística con la profilaxis antibiótica en cesárea con dosis única de Cefazolina respecto a dosis múltiple en Endometritis $(7,14 \%$ vs $1,25 \%, p=0,0628)$, infección de la herida quirúrgica $(9,41 \%$ vs $18,51 \%, p=0,0905)$ e Infección urinaria $(23,52 \%$ vs $21,25 \%, p=0,7265)$. En Nepal, Shakya (20) también encontró que no hubo diferencia significativamente estadística entre el empleo de una y múltiples dosis. 
En nuestro medio, la guía del MINSA (21) del 2007 y la del Instituto Nacional Materno Perinatal (22) publicada en el 2010, recomiendan el uso de profilaxis antibiótica post-clampaje con una dosis de Cefazolina 1 gr EV.

El Hospital Nacional Guillermo Almenara Irigoyen (HNGAl) es uno de los hospitales de máxima referencia del país donde la tasa de cesáreas es del $70 \%$ (23). A pesar de ello, no existe una guía de práctica clínicaacerca del uso de profilaxis antibiótica en procedimientos obstétrico.

La indicación más común, es la administración de 3 dosis de $1 \mathrm{gr}$ de Cefazolina endovenosa en la sala de post-operadas generalmente entre 15 y 120 minutos post-clampaje. Por ello, sería conveniente utilizar un régimen con dosis única que sea tan efectiva como la dosis triple y de este modo disminuir las horas de cuidado, costos de tratamiento, posibilidad de resistencia bacteriana intrahospitalaria y morbilidad.

El objetivo de este estudio fue determinar la efectividad de la dosis única comparada a la dosis triple de Cefazolina como profilaxis antibiótica para la prevención de la infección post-cesárea.

\section{MATERIAL Y MÉTODOS}

\section{Selección de pacientes}

Se realizó un estudio observacional, analítico, retrospectivo, transversal y comparativo en el Servicio de Obstetricia de Alto Riesgo (SOAR) del HNGAI. Se incluyeron todas las historias clínicas de pacientes sometidas a cesárea en el año 2011 que recibieron una o tres dosis de Cefazolina como profilaxis antibiótica. Posteriormente, se establecieron 2 grupos según la dosis recibida:

Dosis única: Pacientes que recibieron una dosis de Cefazolina 1g EV

Dosis triple: Pacientes que recibieron tres dosis de Cefazolina $1 \mathrm{~g}$ EV cada 8 horas.

No se incluyeron aquellas pacientes que presentaron fiebre o infección preoperatoria evidente, trabajo de parto prolongado ( $\geq 12$ horas), ruptura prematura de membranas prolongada ( $\geq 24$ horas), con antecedente de alergia a penicilinas, tratamiento antibiótico dentro de los 7 días previos a la cesárea y/ o uso de corticoides.

\section{Recolección de datos}

Se realizó a través de la ficha de recolección de datos diseñado para este estudio. Todos los datos fueron extraídos de las historias clínicas del SOAR y se revisaron los dos controles posteriores a la cesárea (a los 7 días y al mes). Los datos recolectados incluyeron características sociodemográficas de las pacientes, antecedentes obstétricos, comorbilidades, datos operatorios (indicación, tipo de cesárea, incisión, duración, cirugía adicional, etc.), número de dosis recibida, complicaciones postoperatorias como la estancia operatoria y la presencia de infecciones.

\section{Medición de resultados}

El principal parámetro para comparar la efectividad de ambos esquemas, fueron los datos sugestivos y/ 0 el reporte médico de las siguientes complicaciones:

- Fiebre puerperal: Temperatura $\geq 38^{\circ} \mathrm{C}$ en dos o más determinaciones, separadas por un intervalo mínimo de 4 horas, excluyendo las primeras 24 horas post-parto.

Infección del sitio operatorio (ISO): Infección que ocurre en los 30 días posteriores a la cirugía, o en el plazo de un año si se dejó un implante; afecta piel y tejido celular subcutáneo (ISO incisional superficial), o tejidos blandos profundos de la incisión (ISO incisional profunda) y/ o algún órgano o espacio manipulado durante la intervención (ISO de órganos y espacios) (24).

Endometritis: Fiebre $\left(\geq 38^{\circ} \mathrm{C}\right)$ asociado a uno o más de lo siguiente: dolor uterino, loquios con mal olor, y leucocitosis de $>12,000$ después de la exclusión de otros sitios de infección que desarrolló dentro de los primeros 5 días postparto (25). 
- Infección urinaria (ITU): Urocultivo positivo para un organismo patógeno en un paciente con urocultivo pre-operatorio estéril ó urocultivo positivo para un organismo patógeno diferente en los días previos a la cirugía (26).

- Complicación infecciosa grave: Se consideraran bacteriemia, shock séptico, tromboflebitis séptica, fascitis necrotizante y muerte (3).

\section{Determinación del tamaño muestral}

Se utilizó para su cálculo el programa Epidat 4.0 en su módulo de tamaño de muestra para comparación de proporciones de muestras independientes. Para un poder de prueba de $80 \%$ y nivel de confianza de $95 \%$, se consideró un porcentaje de infección de sitio operatorio de $1.5 \%$ (3)en el grupo de dosis única y en el grupo de dosis triple de 15\% (3).

Se obtuvo un tamaño muestral mínimo de 65 historias clínicas de gestantes sometidas a cesárea para cada grupo lo que hace un total de 130 historias clínicas.

\section{Análisis estadístico}

La información obtenida se analizó mediante el programa SPSS 20.0. Las características generales de la población fueron comparadas entre ambos grupos. Se utilizó la prueba de Chi-cuadrado y se consideró como significante un valor de $\mathrm{p}<0.05$.

\section{RESULTADOS}

En total, 168 gestantes que fueron sometidas a cesárea se incluyeron en el estudio. Se excluyeron 86 pacientes (Figura 1), de las cuales 74 no cumplieron los criterios de inclusión y exclusión, y 12 historias clínicas no contaron con los datos suficientes para efectos de este estudio, quedando finalmente 82 pacientes.

Las características generales de las poblaciones de ambos grupos están descritas en la tabla 1. La edad media de las pacientes incluidas fue de 32 años, teniendo al 65,9\% entre 20 a 34 años de edad.
La mayoría fueron multigestas $(50 \%)$, con una gestación a término $(84,1 \%$ ) y con un adecuado control prenatal (97,6\%). El 70,7\% tenían hemoglobina pre-quirúrgica en valores normales. El $53,6 \%$ de las gestantes tenían el antecedente de cesárea previa.

Figura 1. Fluj ograma de pacientes seleccionados en el estudio

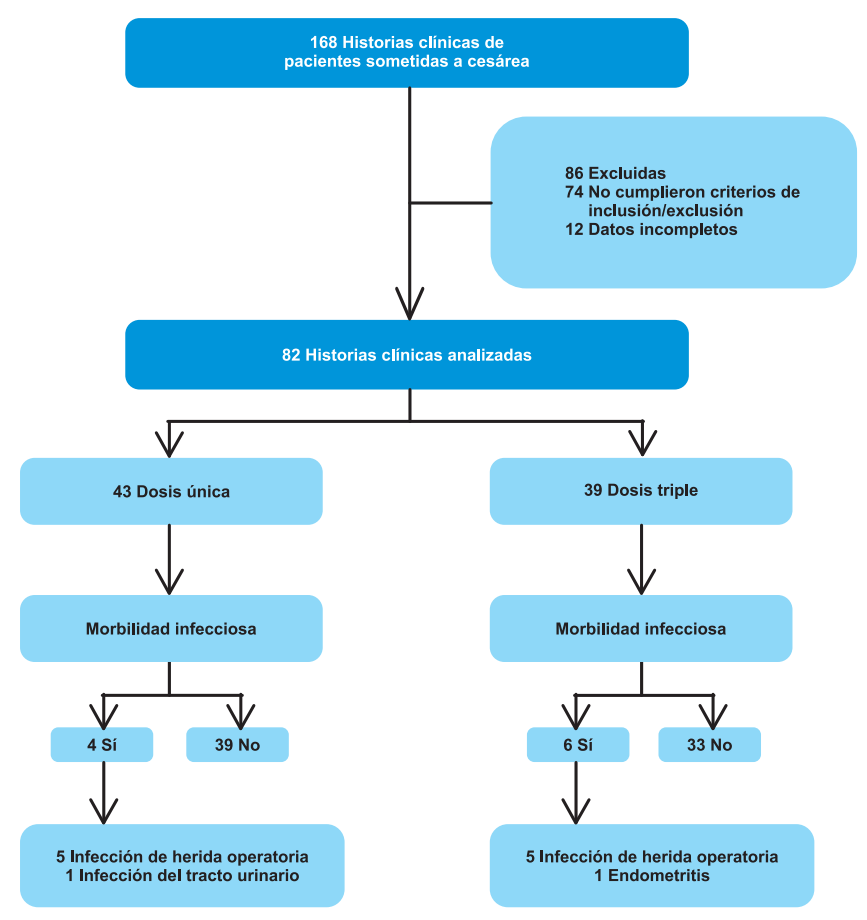

Dentro de las indicaciones de cesárea, la más frecuente para ambos grupos fue la cesárea anterior, con 14 casos en dosis única $(32,6 \%$ y 11 casos en triple dosis $(28,2 \%)$. Tabla 1.

En segundo lugar fue la incompatibilidad feto pélvica con 8 casos $(18,6 \%)$ en dosis única y 10 $(25,6 \%)$ en triple dosis.

Las características operatorias fueron muy similares entre el grupo de dosis única y el de dosis triple. La mayoría de las cesáreas fueron realizadas de manera electiva $(81,4 \%$ vs $79,4 \%)$, y el tipo de incisión aplicada preferentemente fue la transversal $(97,7 \%$ vs $94,9 \%$ ). Por otra parte, la epidural fue el tipo de anestesia más utilizado $(65,1 \%$ vs $53,8 \%)$, aunque la espinal alcanzo un número importante ( $34,9 \%$ vs $43,6 \%)$. 
Tabla 1. Indicación de cesárea

Características

\section{Tipo de cesárea}

Electiva

Emergencia

Incisión

Transversal

Mediana

Anestesia

Espinal

Epidural

General

Cirugía Adicional

Bloqueo tubárico bilateral

Quistectomía

Tiempo operatorio $(\min )$

$<40$

40-55

55-70

$>70$

Pérdida sanguínea ( $\mathrm{ml}$ )

$<500$

500-999

Estancia postoperatoria (media)

En cuanto a la cirugía adicional, prácticamente todas fueron bloqueos tubáricos bilaterales $(25,6 \%$ vs $33,3 \%)$.

Un dato importante es que gran parte de las cesáreas se realizaron sin sobrepasar los 55 minutos (93\% vs $71,8 \%$ ), presentándose además pérdidas sanguíneas dentro de los límites normales $(<500 \mathrm{ml})$ en la mayoría de las pacientes $(74,4 \%$ vs $69,2 \%)$.

Por último, el tiempo de estancia operatoria no sobrepaso los tres días en ninguno de los dos grupos (2,7 días vs 2,8 días).

\section{Dosis Única}

$(n=43)$

\section{Triple dosis}

$(n=39)$

$$
35(81,4 \%)
$$

$8(18,6 \%)$

$42(97,7 \%)$

$1(2,3 \%)$

$15(34,9 \%)$

$28(65,1 \%)$

$11(25,6 \%)$

$16(37,2 \%)$

$24(55,8 \%)$

$2(4,7 \%)$

$1(2,3 \%)$

$32(74,4 \%)$

$11(25,6 \%)$

2,7 días
$31(79,5 \%)$

$8(20,5 \%)$

$37(94,9 \%)$

$2(5,1 \%)$

$17(43,6 \%)$

$21(53,8 \%)$

$1(2,6 \%)$

$13(33,3 \%)$

$1(2,6 \%)$

$13(33,3 \%)$

$15(38,5 \%)$

$10(25,6 \%)$

$1(2,6 \%)$

$27(69,2 \%)$

$12(30,8 \%)$

2,8 días

En el grupo de 43 pacientes a los cuales se les administró dosis única de Cefazolina, el índice de infección de herida operatoria fue del $7 \%$, comparado con un índice del $12,8 \%$ en el grupo de 39 pacientes que recibieron tres dosis del antibiótico.

Se observó que la endometritis se presentó únicamente en el grupo de dosis triple $(2,6 \%)$. Por el contrario, el único caso de infección del tracto urinario fue hallado en el grupo de dosis única.

Finalmente, los resultados no tuvieron una diferencia estadísticamente significativa $(p=0,422)$. 


\section{DISCUSIÓN}

En el Hospital Nacional Guillermo Almenara Irigoyen la tasa de cesáreas llega al $70 \%$ y que es el factor de riesgo más importante de infección materna puerperal, determina un efecto directo en el aumento de la morbilidad materna. Por este motivo, la OMS recomienda la administración de profilaxis antibiótica en las pacientes sometidas a cesárea señalando que el uso de una dosis de profilaxis antibiótica es suficiente y no es menos efectiva que el uso de tres dosis en la prevención de infecciones.

En el presente estudio, la incidencia de morbilidad infecciosa post-cesárea fue de 9,3\% para el grupo de dosis única y de $16,4 \%$ para el de triple dosis, no encontrando diferencia estadísticamente significativa entre ambos (tabla 4).

Estos resultados son similares a los hallados por Gallonet al (19) en donde la tasa de infección para dosis única fue de $34,11 \%$ y de $33,33 \%$ para dosis triple. Similares resultados fueron descritos por Shakyaet alen Nepal (20). La ISO fue la de mayor incidencia, encontrándose en 7 y $12,8 \%$ de los casos de dosis única y dosis triple respectivamente (tabla 4).

Esto podría deberse a que en un $50 \%$ de los casos se realizó bloqueo tubárico bilateral, ya que existe una mayor manipulación y tiempo de exposición del sitio operatorio. Asimismo, nuestros resultados son congruentes con Bagrateeet al (27), quien comparó el uso de Cefoxitina versus un placebo, concluyendo que la infección de herida quirúrgica es la complicación más común post cesárea independientemente de las dosis de profilaxis recibida.

Las otras complicaciones fueron la endometritis y la ITU con una incidencia del $2,3 \%$ en el grupo de dosis triple y $2,6 \%$ en el grupo de dosis única (tabla 4 ). El bajo porcentaje de las infecciones mencionadas se correlaciona con los hallados por Shakyaet al(20), y otros estudios similares. $(19,27,28)$.

Dentro de las indicaciones de parto por cesárea, la más frecuente fue el antecedente de cesárea previa, el cual se presentó en $32,6 \%$ y $28,2 \%$ en los grupos de dosis única y triple respectivamente (tabla 2).

El problema radica en que más de un tercio de las indicaciones fueron por solo una cesárea anterior, lo cual no constituye una indicación absoluta. Es importante mencionarlo debido a que la reducción de infecciones post-cesárea se basa principalmente en la disminución del número de cesáreas innecesarias.

Debido a que se encontraron historias clínicas en donde se utilizaba otro antibiótico o se cambiaba las indicaciones de dosis única de Cefazolina por dosis triple, no se pudo alcanzar el tamaño muestral calculado (65 por cada grupo) para este estudio. Por este motivo, la principal limitaciónfue el reducido tamaño de muestra ya que nos impide generalizar los hallazgos al resto de la población. Otra limitación identificada fue la imposibilidad de evaluar la morbilidad infecciosa a largo plazo, ya que no todos los pacientes acudieron a sus controles. Por otro lado, la fortaleza fue el comportamiento homogéneo y comparable de la mayoría de variables en ambos grupos, lo que demuestra que estas no juegan un papel importante en la aparición o no de infección.

En conclusión, la dosis única de Cefazolina fue igualmente efectiva a la dosis triple en la prevención de las complicaciones infecciosas post-cesárea al no existir diferencia estadísticamente significativa.

Es decir, mayores dosis no aumentan la eficacia de la protección. Ambos regímenes fueron seguros y bien tolerados con ningún evento inesperado. Sin embargo, el empleo de una dosis reduciría los costos, la toxicidad, la resistencia bacteriana y los errores en la administración, razón por la cual esta sería la mejor opción como tratamiento antibiótico profiláctico en las cesáreas.

\section{Agradecimiento}

Al Dr. Luis Guerra Diaz, Gineco-Obstetra del Servicio de Alto Riesgo Obstétrico del Hospital Nacional Guillermo Almenara Irigoyen, por incentivarnos a realizar el tema de investigación. 


\section{Fuentes de financiamiento}

\section{Este estudio ha sido autofinanciado por los autores.}

\section{Conflictos de interés}

\section{Los autores declaran no tener ningún conflicto de interés.}

\section{REFERENCIAS BIBLIOGRÁFICAS}

1. Gibbs RS. Clinical risk factors for puerperal infection. Obstet Gynecol. 1980; 55: 178-183.

2. Declercq E, Barger M, Cabral HJ, Evans SR, Kotelchuck M, et al. Maternal outcomes associated with planned primary cesarean births compared with planned vaginal births. Obstet Gynecol. 2007 Mar; 109(3):669-77.

3. Smaill F, Hofmeyr GJ. Profilaxis antibiótica para la cesárea. Base de Datos Cochrane de Revisiones Sistemáticas 2002, Issue 3.

4. Steer JP. Puerperal sepsis. In: Chamberlain G, Steer P, editors. Turnbull's Obstetrics. 3rd ed. London: Churchill Livingstone; 2001. p.663-70.

5. Betrán AP, Merialdi M, Lauer JA, Bing-Shun W, Thomas J, et al. Rates of caesarean section: analysis of global, regional and national estimates. PaediatrPerinatEpidemiol. 2007; 21 (2):98.

6. Betrán AP, Gulmezoglu AM, Robson M, Merialdi M, Souza JP, et al. WHO global survey on maternal and perinatal health in Latin America: classifying caesarean sections. Reprod. Health. 2009; $6: 18$.

7. Smaill FM, Gyte GM. Antibiotic prophylaxis versus no prophylaxis for preventing infection after cesarean section. Cochrane Database Syst. Rev. 2010

8. Enkin MW, Enkin E, Chalmers I, Hemminki E. Prophylactic antibiotics in association with caesarean section. In: Chalmers I, Enkin MW, Keirse MJNC editor(s). Effective Care in Pregnancy and Childbirth. Oxford: Oxford UniversityPress, 1989: 124669.

9. Chelmow D, Ruehli MS, Huang E. Prophylactic use of antibiotics for nonlaboring patients undergoing cesarean delivery with intact membranes: a meta-analysis. Am J Obstet Gynecol. 2001; 184: 656-61

10. Alfirevic Z, Gyte GM, Dou L. Different classes of antibiotics given to women routinely for preventing infection at caesarean section. Cochrane Database SystRev. 2010

11. Faro S, Martens MG, Hammill HA, et al. Antibiotic prophylaxis: is there a difference? Am J Obstet Gynecol. 1990; 162:900.

12. Committee opinion no. 465: Antimicrobial prophylaxis for cesarean delivery: timing of administration. Obstet Gynecol. 2010; 116(3):791.

13. Hopkins L, Smaill F. Antibiotic prophylaxis regimens and drugs for cesarean section. Cochrane Database Syst Rev. 1999, Issue 2.

14. Schalkwyk JV, et al. Antibiotic Prophylaxis in Obstetric Procedures. J Obstet Gynaecol Can (SOGC). 2010;32(9):878-884
15. Mangram AJ, Horan TC, Pearson ML, Silver LC, Jarvis WR. Guideline for prevention of surgical site infection, 1999. Centers for Disease Control and Prevention (CDC) Hospital Infection Control Practices Advisory Committee. Am J Infect Control. 1999 (27): 97-134.

16. Gordon SM. Antibiotic prophylaxis against postoperative wound infections. Cleve Clin J Med. 2006; 73(1): 42-45.

17. ACHS. Clinical Indicator User Manual 2012.

18. The Royal Australian and New Zealand College of Obstetricians and Gynaecologists. NEW College Statement C-Gen 17. 2011 Nov.

19. Gallón LJ, Gonzales AM, Restrepo L, Agudelo LM. Dosis única vs dosis múltiple de Cefazolina como profilaxis antibiótica en cesáreas no electivas. Revista CES Medicina. 1996; 10(2).

20. Shakya A, Sharma J. Comparison of Single versus multiple doses of antibiotic prophylaxis in reducing post-elective Caesarean section infectious morbidity. Kathmandu Univ Med J. 2010; 8(2): 179-184.

21. MINSA. Guías de Práctica Clínica para la Atención de Emergencias Obstétricas según nivel de Capacidad Resolutiva. 2007. Perú.

22. Instituto Nacional Materno Perinatal. Guías de Práctica Clínica y de Procedimientos en Obstetricia y Perinatología. 2010. Perú.

23. Servicio de Obstetricia de Alto Riesgo. Informe anual de partos atendidos por clase de parto. 2011. Hospital Nacional Guillermo Almenaralrigoyen - Essalud.

24. Vilar D, García B, Sandoval S, Castillejos A. Surgical site infections. From the pathogenesis to prevention. Enf Inf Microbiol. 2008; 28 (1): 24-34.

25. Ziogos E, Tsiodras S, Matalliotakis I, Giamarellou H, Kanellakopoulou K. Ampicillin/Sulbactam versus Cefuroxime as antimicrobial prophylaxis for cesarean delivery: a randomized study. BMC Infect Dis. 2010; 10:341.

26. Alekwe LO, Kuti O, Orji EO, Ogunniyi SO.Comparison of ceftriaxone versus triple drug regimen in the prevention of cesarean section infectious morbidities. J Matern Fetal Neonatal Med. 2008 Sep; 21(9): 638-642.

27. Bagratee JS, Moodley J, Kleinschmidt I, Zawilski W. A randomized controlled trial of antibiotic prophylaxis in elective caesarean delivery. Br J Obstet Gynaecol. 2001;108:143-148.

28. Sullivan A, Smith T, Chang E, Hulsey T, Vandorsten JP, et al. Administration of cefazolin prior to skin incision is superior to cefazolin at cord clamping in preventing postcesarean infectious morbidity: a randomized, controlled trial. Am J Obstet Gynecol. 2007;196;455.e1-455.e5.

\section{Correspondencia:}

\section{Pedro Raul Ochoa Allemant}

Dirección: Av Paul Linder 349, San Borja. Lima, Perú

Teléfono: +51975008836

Correo electrónico: pedro.ochoa.allemant@hotmail.com

Recibido: 24 de J ulio de 2014

Aprobado: 29 de Agosto de 2014 